chinesischer Kinder in Taiwan, Hong Kong und den U.S.A. vor. White befaßt sich, gestützt auf Material aus den 50er Jahren, mit der Einwohnerregistrierungspflicht in chinesischen Städten als eines Mittels der sozialen Kontrolle. Bennett kommt bei der Untersuchung der Funktion chinesischer Massenbewegungen bei der Sozialisation $\mathrm{zu}$ dem erstaunlichen Schluß, die soziale Herkunft von Individuen wirke sich auf deren jeweilige Beteiligung an Massenbewegungen nicht aus. Bloom hat Abstraktionsfähigkeit und Originalität im soziopolitischen Denken Hong Konger Chinesen empirisch untersucht und überträgt seine Ergebnisse recht spekulativ auf die Situation in der Volksrepublik. Der Ókonom Hoffmann schildert in Fortführung einer 1967 erschienenen Studie die Mechanismen des Arbeitsanreizes in unterschiedlichen Phasen der Geschichte der Volksrepublik China - ein gerade durch die nachmaoistische Entwicklung (zu der Hoffmann sich noch nicht äußert) wieder hochaktuell gewordenes Thema. J. Huang, Literaturwissenschaftler, umreißt die Figur der ,,bösen Frau" in der revolutionären Literatur Chinas, dabei das Fortdauern traditioneller chinesischer Moralvorstellungen betonend. Munro erörtert den philosophischen, insbesondere ethischen Hintergrund des ,, belief control“ im modernen China; auch er geht auf traditionelle Wurzeln ein und bemüht sich im übrigen, Unterschiede zur sowjetischen Denkungsweise herauszuarbeiten, die dem Gedanken einer ,freien“ Willensentscheidung aufgeschlossener gegenüberstünde als die moderne chinesische. Die Abwesenheit eines Rechtswissenschaftlers bei der vorbereitenden Konferenz haben die Herausgeber durch den Nachdruck eines kurzen Beitrages von Victor Li aus dem Jahre 1973 über Strafrecht in der Volksrepublik China auszugleichen versucht.

Das Fehlen eines detaillierten Konzeptes, das die einzelnen Beiträge hätte strukturieren können sowie auch eines die Einzelergebnisse zusammenordnenden Resümees ist gewiß bedauerlich; gleichwohl sind die Beiträge durchweg lesenswert. Die Bearbeitung der bislang noch wenig behandelten Fragen der sozialen Kontrolle im China von heute wird in Zukunft ganz sicher von der breiteren empirischen Basis profitieren, die die Öffnung der chinesischen Sozialwissenschaften für Kontakte mit dem Ausland zu vermitteln verspricht. Ein nicht allein dieses Buch, sondern einen großen Teil insbesondere US-amerikanischer Literatur der letzten Jahre treffendes Wort der Kritik sei schließlich noch angefügt: Manche teure Seite könnten Verlage zum Nutzen der Leser einsparen, wenn sie geschwätzige Berichte über Freud und Leid der Autoren bei der Vorbereitung, nicht endenwollende Danksagungen für jede Handreichung und Feststellungen wie diejenige unterbänden, daß die Konferenzteilnehmer freudig einen Teil (!) der Kosten für die Mahlzeiten selbst gezahlt haben (,,most cheerfully“").

Philip Kunig

\title{
Romain YAKEMTCHOUK
}

\section{La Convention de Lomé,}

Nouvelles formes de la coopération entre la C.E.E. et les Etats d'Afrique, des Caraîbes et du Pacifique. Koninklijke Academie voor Overzeese Wetenschappen, Brüssel, 1977, 181 S. (nicht im Handel).

Die Konvention von Lomé 1 , abgeschlossen im Februar 1975 und in Kraft getreten im April 1976, regelt die Wirtschaftsbeziehungen zwischen den neun Staaten der Europäischen Wirtschaftsgemeinschaft und (inzwischen) 53 Staaten Afrikas sowie aus dem karibischen und pa-

1 BGBl. 1975 II, S. $2317 \mathrm{ff}$. 
zifischen Raum (im EG-Jargon als AKP-Staaten bezeichnet). Sie enthält Bestimmungen über Zollpräferenzen sowie über finanzielle und technische Zusammenarbeit und errichtet das als STABEX bekannte System zur Stabilisierung der Exporterlöse in bestimmten Rohstoffbereichen (zwölf Produkte). Insbesondere der Absatz ihrer tropischen Produkte (die in keinem Konkurrenzverhältnis zu den Erzeugnissen des EG-Agrarmarktes stehen) ist für die AKP-Staaten infolge der Konvention leichter als für andere Entwicklungsländer. Bemerkenswerte im Hinblick auf die wirtschaftspolitische Souveränität ist die Verpflichtung der AKP-Staaten, für ein günstiges Investionsklima sorge zu tragen. Die vorliegende Studie stellt die Konvention in ihren historischen, insbesondere rechtshistorischen Zusammenhang, in dem sie zunächst die Probleme der Assoziierung überseeischer Territorien von deren Unabhängigkeit ins Gedächtnis ruft, sich sodann vor allem den Beziehungen zwischen Belgien und dem Kongo sowie Ruanda-Urundi zuwendet (Dieser Abschnitt stellt eine gestraffte und überarbeitete Fassung des entsprechenden Kapitels aus einem früheren Werk des Autors dar². Yakemtchouk schildert dann die Entwicklung des Assoziationskonzeptes von seinen Anfängen in den Römischen Verträgen bis hin zum Abschluß der Konvention von Lomé und erläutert ausführlich und sorgfältig deren Mechanismen. Der Aspekt der Bürokratisierung durch die Errichtung eines Netzes von ,,Euro-Botschaften“ mit missionsähnlichem Status, die allein im afrikanischen Bereich im Jahr 1977 rund 50 Millionen Schweizer Fanken verschlungen haben ${ }^{3}$; findet dabei nicht die Berücksichtigung, die er verdient.

Der Autor erhebt nicht den Anspruch, die Konvention von Lomé entwicklungspolitisch einordnen zu wollen, widmet sich vielmehr in der Hauptsache der Beschreibung. Sobald er dieses Feld verläßt, werden seine Aussagen zum Teil bezweifelbar. So sieht Yakemtchouk die Konvention als Ausdruck einer Bewußtwerdung des Problems der Unterentwicklung bei ,,den Völkern Europas“ an, einer solidarité humaine, die die nationalen Egoismen überwunden haben (S. 9, S. 174 f.). Dieser Sicht der Dinge gegenüber ist darauf hinzuweisen, daß die Konvention den Verlauf der Handelsströme, wie er in der kolonialen Epoche entstanden ist, kaum verändert hat. Auch wenn sie insgesamt ,,egalitärer“ angelegt ist als ihre Vorgängerin, die Yaounde-Konvention, spricht wenig dafür, die Konvention von Lomé als ,,Schlußpunkt der Dekolonialisierung " zu feiern", und viel, sie als Versuch der Stabilisierung des herkömmlichen Weltwirtschaftssystems anzusehen ${ }^{5}$. Zurecht merkt Yakemtchouk an, daß auch das Lomé-System nur vorläufigen Charakter habe (S. 173); über die Tendenz der künftigen Modifikationen kann er sich noch nicht äußern (das Manuskript wurde offenbar Mitte 1976 abgeschlossen). Die derzeit in Gang befindlichen Verhandlungen über „,Lomé II“, die nach Vorstellungen der EG einen umfassenden Konsultationsmechanismus betreffend jede Investitionsentscheidung einrichten sowie die Einhaltung europäisch definierter Menschenrechtsstandards durch die AKP-Staaten durchsetzen sollen ${ }^{6}$, werden letztlich erweisen, inwieweit die optimistische Darstellung Yakemtchouks gerechtfertigt ist - ob der von der Konvention von Lomé eingeschlagene Weg tatsächlich dazu führen kann, daß neben die völkerrechtliche Unabhängigkeit der Entwicklungsländer auch mehr wirtschaftliche und politische Selbstbestimmung treten kann.

Philip Kunig

\footnotetext{
2 L'Afrique en droit international, 1971.

3 NZZ vom 23. Dezember 1977, S. 11.

4 So aber in dieser Zeitschrift D. Schröder (VRU 10, 1977, S. 131).

5 Vgl. z. B. Galtung, The AfricanReview 6 (1976), S. 33 ff., sowie Tezlaff, VRU 9 (1976), S. 49 ff. m. w. Nachw.; s. auch die Beiträge in Heft 3-4/1978 der Entwicklungspolitischen Korrespondenz.

6 Vgl. Bulletin der EG Nr. 2/1978 Ziff. 1.3.4.
} 